\title{
Automated local line rolling forming and simplified deformation simulation method for complex curvature plate of ships
}

\author{
Yao Zhao ${ }^{1,2}$, Changcheng Hu${ }^{1}$, Hongbao Dong ${ }^{1}$, and Hua Yuan ${ }^{1}$ \\ ${ }^{1}$ School of Naval Architecture and Ocean Engineering, Huazhong University of Science and Technology, \\ Wuhan, 430074, P. R. China \\ ${ }^{2}$ Collaborative Innovation Center for Advanced Ship and Deep-Sea Exploration (CISSE), \\ Shanghai, 200240, P. R. China \\ Correspondence to: Yao Zhao (yzhaozzz@ hust.edu.cn)
}

Received: 5 November 2016 - Revised: 11 March 2017 - Accepted: 9 May 2017 - Published: 2 June 2017

\begin{abstract}
Local line rolling forming is a common forming approach for the complex curvature plate of ships. However, the processing mode based on artificial experience is still applied at present, because it is difficult to integrally determine relational data for the forming shape, processing path, and process parameters used to drive automation equipment. Numerical simulation is currently the major approach for generating such complex relational data. Therefore, a highly precise and effective numerical computation method becomes crucial in the development of the automated local line rolling forming system for producing complex curvature plates used in ships. In this study, a three-dimensional elastoplastic finite element method was first employed to perform numerical computations for local line rolling forming, and the corresponding deformation and strain distribution features were acquired. In addition, according to the characteristics of strain distributions, a simplified deformation simulation method, based on the deformation obtained by applying strain was presented. Compared to the results of the three-dimensional elastoplastic finite element method, this simplified deformation simulation method was verified to provide high computational accuracy, and this could result in a substantial reduction in calculation time. Thus, the application of the simplified deformation simulation method was further explored in the case of multiple rolling loading paths. Moreover, it was also utilized to calculate the local line rolling forming for the typical complex curvature plate of ships. Research findings indicated that the simplified deformation simulation method was an effective tool for rapidly obtaining relationships between the forming shape, processing path, and process parameters.
\end{abstract}

\section{Introduction}

At present, forming of the complex curvature plate for ships can be classified into mechanical cold forming and thermal forming from the viewpoint of the loading method. From a process viewpoint, it is divided into local line loading forming and monolithic loading forming. In detail, the line loading here does not signify that the loading comes into play on an identical line of the plate, but makes the point load exert continuous actions on a certain line in order. As far as the current shipbuilding enterprises are concerned, line heating and mechanical cold press forming are often used for manufac- turing these curvature plates. In line heating, the shrinkage strain generated during heating and cooling in some parts of the metal plate causes deformation of the plate. With regard to such a line heating forming approach, Ueda et al. (1991, 1993, 1994a, b) predicted line heating forming paths based on the inherent strain calculation. And Nguyen et al. (2009) used artificial neural networks to carry out similar studies. Based on theoretical studies, automation equipment adopting the line heating forming approach has been successfully developed so that the processing efficiency for hull plates can be effectively improved (Yoshihiko et al., 2011). Nevertheless, owing to the characteristics of the line heating forming 
approach, such as small thermal strains incurred by line heating, the shape obtained through line heating forming is still under restrictions to a certain extent for thicker plates and regions with a larger curvature (Yoshihiko et al., 2011). Correspondingly, the cold mechanical press forming utilizes mechanical devices to apply a bending load to realize plate deformation. Compared to line heating forming, it has a higher efficiency in processing thick plates with a large curvature. Therefore, it is clear that the cold mechanical press forming has its own advantages. However, it is rather difficult to apply the mold forming method, a commonly used cold mechanical press forming method, for the automated molding of complex curvature plates in batches, with considerable differences in shapes and sizes. If variable mold molding is used, Hwang et al. (2010) performed studies on springback control and deviation compensation for multi-indenter molding and developed a multi-indenter molding device. Shim et al. (2011) studied, the mechanism of complex curvature plate molding based on an adjustable punch increment. In addition, they also investigated the use of a multi-roller forming device to realize complex curvature plate forming. Another representative mechanical cold press forming method is the die-free local forming method. In this method, complex curvature plate forming is ultimately realized by constituting a local small-range plastic processing area on the premise that the processing plate enters such an area in diverse orders and from various parts. However, the processing path determination and forming shape control in the above mechanical cold pressing process during contour machining of complex curvature planking for ships still mainly depend on artificial experience.

Clearly, the die-free local forming method mentioned above is very suitable for shipbuilding applications. The local line rolling forming is one of the die-free local forming methods commonly used (see Fig. 2) and has been extensively applied in shipyards. The corresponding basic approach can be described as follows. A pair of concave-convex wheels is mounted onto the upper and lower surfaces of the processing plate to drive the relative up and down motions of the upper and lower wheels. Therefore, local pressure on the processing plate can be realized. Meanwhile, the upper convex wheel or the lower concave wheel drives the motion of the processing plate that further passes between the upper and lower wheels through rotation to realize local bending of the plate. Based on the motions of the concave-convex wheels on the processing plate along with paths in diverse directions, complex curvatures of various shapes can be generated for the plate. From the perspectives of the upper and lower concaveconvex wheels as well as the processing plate, the sphere of loading actions from those wheels is relatively narrower if compared with the length-width dimension of the plate. Moreover, their loading actions can be deemed as a process that approximates rolling line mechanical rolling, which is referred to as the local line rolling forming method here. Similar to the line heating plate forming method, it also involves gradual forming; the overall shape is realized based on the control over local deformation. Such a forming method requires small equipment dimensions and low costs. In this machining process, the processing path and loading force of the roller should be primarily determined according to operator experience. Finally, forming of the target complex curvature plate is completed. In fact, the processing mode based on artificial experience is in line with special knowledge systems. Theoretically speaking, through rational path planning and loading force settings, forming of complex curvature plates with diverse sophisticated shapes can be realized. If an inherent mapping relationship expression involving the forming shape and processing parameters, such as loading path and loading force, that can be accepted by a computer system is developed, a rolling scheme can be accurately formulated. Then, by virtue of the corresponding processing equipment, automation of local line rolling forming can be realized.

During manual operation, the operator determines the preliminary loading magnitude and path according to his/her experience as well by estimating the unloading elastic resilience of the plate in the first place. Second, comparisons between the processing plate after rolling forming and the target shape are carried out by using the cardboards. Third, according to his/her experience, both the rolling feed and rolling path are modified to obtain the target shape by repeating comparisons and modifications. The plate is deformed into the target shape with complex curvature from its initial form, which is mainly caused by uneven in-plane and bending strains inside the plate (Liu and Yao, 2005). After obtaining the in-plane and bending strain distributions required to form the target shape, a processing technique able to exert such strains can be found. These are the general considerations for plate forming processes. Till date, relevant studies in this regard have emphasized the following three issues:

i. an application path of evenly dispersed in-plane or bending strain incurred by the in-plane and bending strain distributions of continuous and uneven target shapes;

ii. finding the corresponding relation between process parameters and in-plane or bending strains;

iii. obtaining a successive correction method for the applied strain approximate to the target shape.

These issues are the focus of this study. With the objective of resolving these problems, experiential knowledge stored in the human brain should be integrally transformed into driving data for automation equipment; plate forming as a sophisticated nonlinear problem should be solved completely using theoretical analysis or experimental measures. However, it is almost impossible to execute these approaches. Numerical calculations with the help of a computer become an inevitable choice. It is feasible to compute different loading paths and loading forces through computer-based numer- 
ical calculations to acquire the corresponding relation between them and the in-plane and bending strains as well as deformation shapes. Further, the numerical computation for the local line rolling forming process involves complicated factors such as contact variations, moving loading, unloading resilience, material type and geometric nonlinearity, if the three-dimensional elastoplastic finite element method is adopted. The time and capacity required for computation are very high In practice, it is also very difficult to complete formation of massive driving data supporting automated forming system for the complex curvature plates of ships. Based on the above discussions, to constitute such driving data, a calculation method with high precision and efficiency should be found, and this is an inevitable link on the entire development cycle.

In this paper, direct at the above local line rolling forming method and for obtaining a highly precise and efficient calculation method, the three-dimensional elastoplastic finite element method is studied for the single local line rolling by taking moving loading, contact point variation, elastoplastic deformation, and unloading resilience into consideration first. Therefore, detailed strain and deformation fields are obtained. In addition, after analyzing the distribution characteristics of the corresponding in-plane and bending strains, a simplified deformation simulation approach acquired for deformation is put forward in allusion to strain application. Then, based on the three-dimensional elastoplastic finite element method, deformations obtained by one- and two-local line rolling are compared relative to their computational accuracies and time. On this basis, the possibility of employing this method to determine the typical complex curvature plate shape in multi-local line rolling is examined. Moreover, with the objective of obtaining relationships between the simplified deformation simulation method and deformation shape, the possibility of utilizing such a method to rapidly conduct a large number of deformation simulation calculations based on the local line rolling forming is verified under conditions of diverse loading paths and forces.

\section{Overall scheme design of local line rolling forming}

The core of the automated local line rolling forming of the complex curvature plate for ships is the formation of driving data. Concrete formation procedures of such driving data are shown in Fig. 1; the connotations of the key points (i) to (iii) mentioned above, corresponding to the formation framework of driving data, are also contained.

Focusing on the key points (i) to (iii) described above, the relationship between the automated local line rolling forming method for the complex curvature plates of ships and deformation simulation calculation method is demonstrated.

Key point (i) pertains to the determination of the local line rolling forming loading path. Here, plate forming mainly concerns both plate deformation and the strain distribution closely related to it (that is the geometrical relationship of plate). Therefore, the large deformation elastic finite element method can be adopted to implement mechanical calculations for the target shape to further obtain deformation and strain distributions. Generally, for convenience, all strain components acquired through calculations are synthesized into the principal strain. Then, according to the definitions of in-plane and bending strains, the in-plane and bending strain distributions represented by the principal strain can be obtained. From the above process, it was found that the acquired strain distributions specific to complex curvature shapes were not only continuous, but also uneven. With regard to the practical process in local line rolling forming, the line-to-line disperse loading is applied to the processing plate in reality. Obviously, the continuity of strain cannot be guaranteed. Seen from another perspective, during practical processing, it is very hard to realize constant changes in the press amount in the process of one line loading owing to the related equipment. In other words, the unevenness of strain cannot be satisfied as well. Therefore, direct at the practically continuous and uneven strain distributions on the target shape plate, a multi-line dispersion loading scheme with invariant strain levels can be generated approximately for a single loading path by formulating a strain accepting/rejecting principle or by integration. In addition, studies in this regard are still under way (Keisuke et al., 2012; Park et al., 2016a, b). Considering that this is not the focus of this study, it will not be described in detail. However, the approximation degree of such a strain-loading path acquired in this manner or the rationality of the accepting/rejecting principle mentioned above should be explored and verified by simulations and calculations.

Key point (ii) states that the corresponding relation between the strain and process parameters should be determined. As far as the local line rolling forming loading is concerned, the principal process parameter is the press amount loaded by the upper/lower wheel. Under practical processing, as described above, the load is exerted in the form of a line and intervals exist between lines. Moreover, for a single loading line, its press amount usually remains unchanged. As a result, the corresponding relation between the strain and processing parameters can be acquired using the three-dimensional elastoplastic finite element method under the condition of a single loading line with diverse press amounts. Despite such a calculation conducted for a single loading line, it is difficult to fully determine the relationship corresponding to a single strain component regardless of process parameter changes, because such parameters have a rather broad variation range. The relationship between the technological parameters and proportion occupied by a certain strain component can be determined. Relational data for technological parameters provided with a small matching difference in the loading paths of key point (i) are presently still obtained through numerical calculations (Liu and Yao, 2005; Shi et al., 2013). However, if their acquisition is com- 


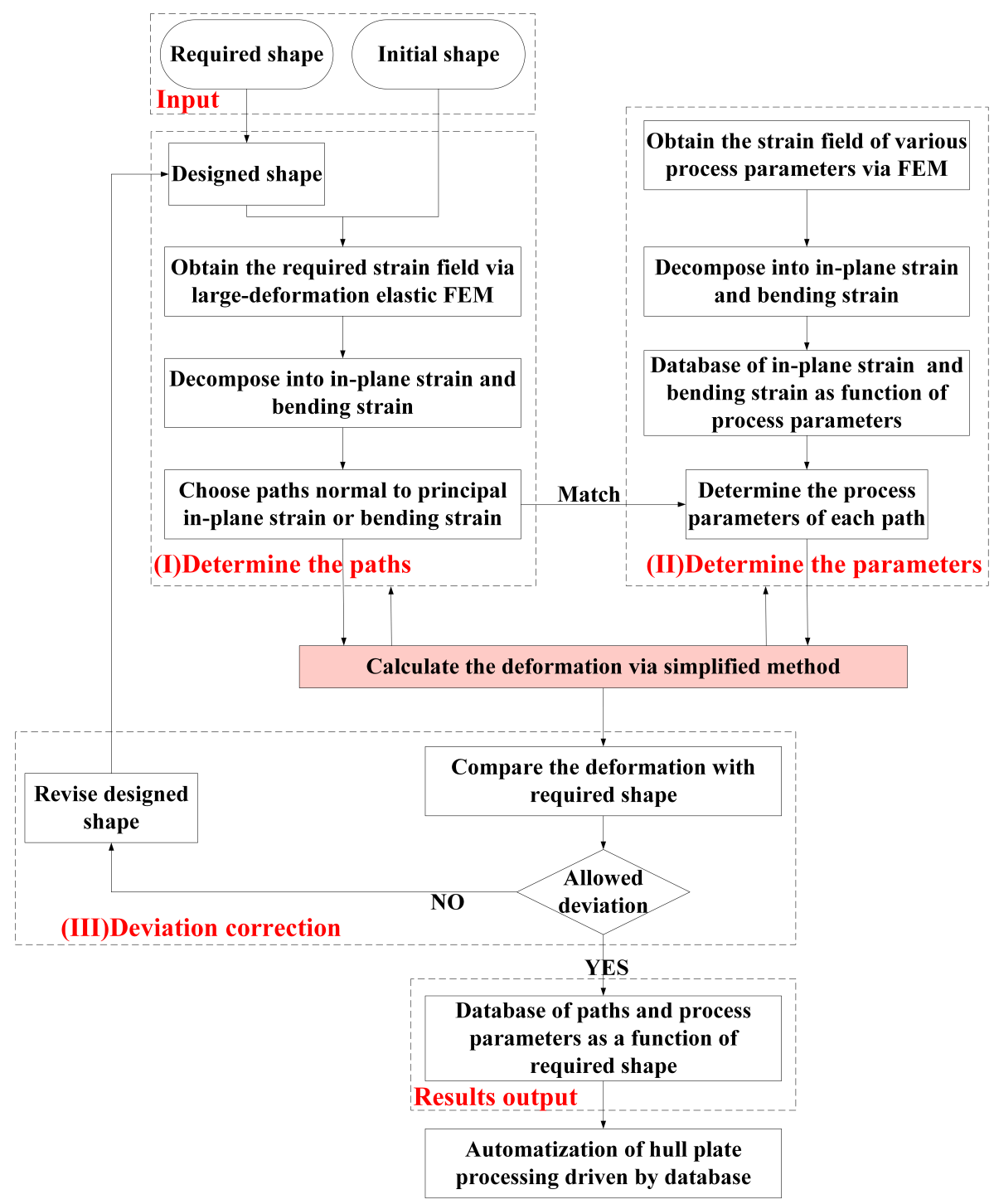

Figure 1. Formation procedure for the automated local line rolling forming driving data of complex curvature plates for ships.

pletely dependent on the three-dimensional elastoplastic finite element method, considerable computational efforts will be required.

For key point (iii), through repeated iterative correction computations for the target shape, the error between (i) and (ii) can be eliminated to satisfy the accuracy requirement of processing driving data. As such an error that cannot be erased easily, it may be confirmed that the application of (ii) into (i) fails to meet the accuracy demand of the target shape. Here, relying on the results of key points (i) and (ii), the difference between the target shape and the numerical forming outcome can be discovered by numerical calculation. Then, this difference is adopted to modify the target shape, so that the designed shape can be obtained. Subsequently, the strain distribution and the corresponding loading path and process parameters are all obtained, followed by the implementation of new numerical forming calculations and accuracy verification. Based on these iterative loop calculations, the required precision for the target shape can be satisfied. In particular, if the numerical forming calculations are performed using the three-dimensional elastoplastic finite element method, it is difficult to imagine how significant the computational efforts and how low the computational efficiency will be.

Figure 1 indicates not only the relation between the automated local line rolling forming driving data and the key points, but also the relevance of a highly precise and efficient computational method. The simplified deformation simulation method proposed in this study plays an important role throughout the forming process. 


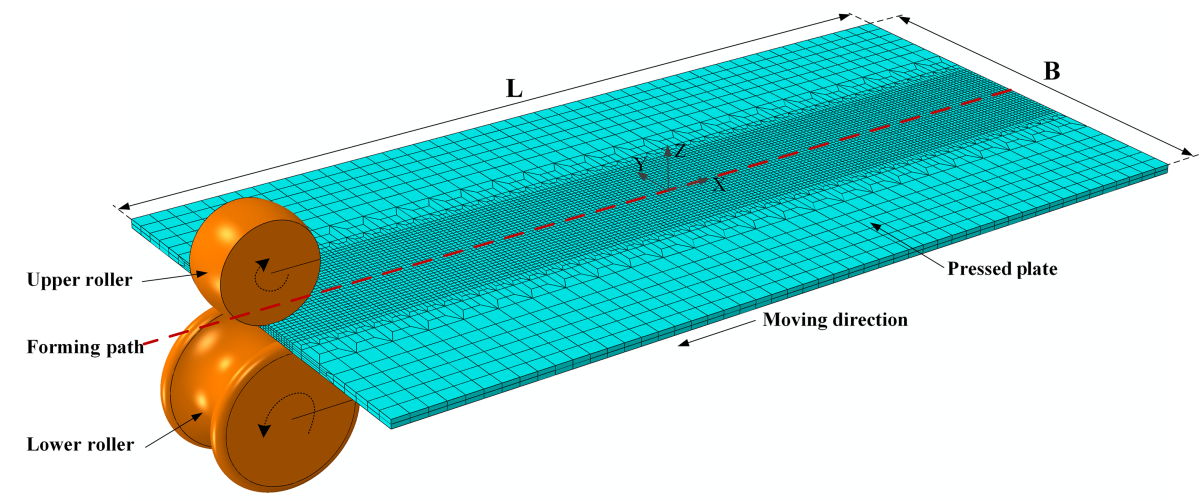

Figure 2. Finite element model and mesh generation for local line rolling forming.

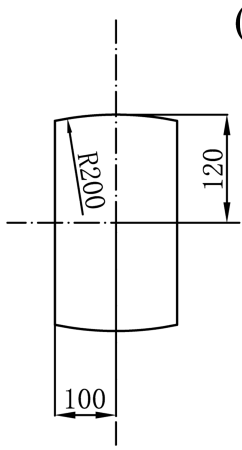

(a)

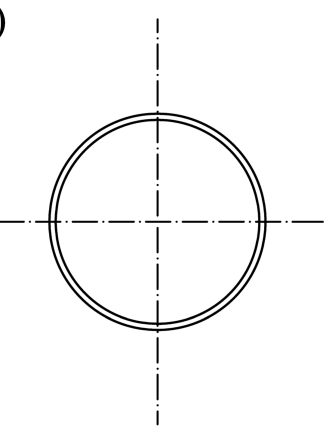



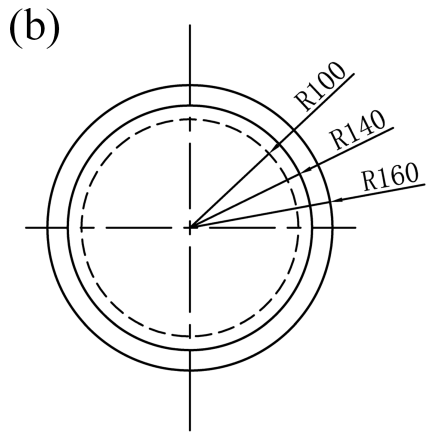

Figure 3. Shape and dimensions of upper/lower roller (unit: mm). (a) Upper Roller. (b) Lower Roller.

\section{Model and results of elastoplastic finite element analysis}

To understand the mechanical process of local line rolling forming in a comprehensive manner and lay a foundation for the presentation of a simplified deformation simulation method, three-dimensional elastoplastic finite element simulations and result analyses were first carried out for local line rolling. As the local line rolling forming is a discrete line loading performed on the pressed plate, it is assumed that a certain distance exists between lines. In other words, mechanical quantity variations caused by one loading line have little influence on another loading line. For this study, a specific three-dimensional elastoplastic finite element method simulation was carried out direct at the local line rolling forming under the action of a single loading line in the first place.

As for local line rolling forming, there exist multiple nonlinear problems such as large deformations, material elastoplasticity, and contact point variation. Therefore, the commercial finite element software ABAQUS was chosen to simulate the three-dimensional elastoplastic finite element calculation. In line with the finite element simulation calculation for plastic forming, ABAQUS/Explicit results were imported into ABAQUS/Standard for springback analyses following calculations based on ABAQUS/explicit (You et al., 2014). The reason is that ABAQUS/Explicit provides a higher computational efficiency than other simulation calculation methods for the forming process. However, it is not suitable for the springback process simulation (Shaohui et al., 2012). In this study, the relative position between the roller and processed plate keeps changing during rolling forming so that the processed plate moves in and out of the rolling area. Furthermore, this process is always accompanied by the forming and springback phenomena. Considering that ABAQUS/Explicit is not suitable for springback calculations, ABAQUS/Standard is employed in this study to perform the numerical simulation of rolling forming.

The geometrical shapes and dimensions of the rollers are shown in Fig. 3. The dimensions of the plate are $2000 \mathrm{~mm} \times 1000 \mathrm{~mm} \times 20 \mathrm{~mm}$. All of the rollers in the model are assumed rigid and are modeled as analytical rigid surfaces, which do not need to be meshed. The finite element mesh for the plate is S4R, which is a four-node, doubly curved, quadrilateral shell element; it includes large rotations, transverse shear deformations, and finite membrane strains. The S4R mesh is formulated for large strains and deformations. To increase the computation speed, the isoperimetric S4R elements were evaluated using reduced integration with one integration point per element, and effective 
hourglass control techniques were used to avoid the spurious deformation modes during finite element simulations. Figure 2 shows the finite element meshing of the plate model; the finite element model total contains 29200 elements and 37067 nodes. The initial elemental dimensions in the plastic area near the loading path are $10 \mathrm{~mm} \times 10 \mathrm{~mm}$ in the plate plane.

Contact between the roller and plate is constrained by a penalty function method. In other words, the external surface of the roller is set as the master surface, while upper and lower surfaces are its slave surfaces. Moreover, interactions exist between the roller and plate in the case where the normal pressure during forming is positive; in comparison, separated rollers fail to interact with each other when the normal pressure is zero or negative. Under the condition that there are interactions between two contact surfaces, the corresponding contact status is defined as finite sliding.

The material parameters of the plate used in the numerical simulation are presented in Table 1. As mentioned above, it is not necessary to endow the roller with material properties because it makes use of an analytic rigid model. At the time of numerical simulation, the plate material is considered isotropic, and it conforms to the Von Mises yield criterion.

Finite element simulation is based on load increment calculation, with each simulation process divided into multiple load increments. The finite element simulation in this study involves the following loading steps:

- Step 1: assemble the components (plate and rollers) according to their geometrical relationship (Fig. 1), and set the contact surface and contact properties between the processed plate and rollers.

- Step 2: apply an artificially set $z$-axial negative minimum displacement load to the upper roller, with the lower roller location unchanged, and carry out the numerical simulation by load increment computation, to establish contact between the rollers and the processed plate.

- Step 3: the upper roller applies an appropriate displacement load to the plate and causes local deformation of the plate.

- Step 4: apply an angular displacement load to the lower roller with the location of the upper and lower rollers unchanged. The contact friction leads to a change in the relative location of the plate and rollers, and the plate gradually enters the rolling zone. In this process, the plate keeps entering and exiting the rolling zone, accompanied by forming and springback. After the completion of rolling on the entire forming path, the upper and lower rollers are removed gradually away from the plate. The contact action between the rollers and plate gradually decreases in this process, and the plate springs back as well, until it is completely separated from the
Table 1. Material parameters.

\begin{tabular}{lcccc}
\hline $\begin{array}{l}\text { Young's } \\
\text { modulus } \\
(E)\end{array}$ & $\begin{array}{c}\text { Poisson's } \\
\text { ratio } \\
(v)\end{array}$ & $\begin{array}{c}\text { Yield } \\
\text { stress } \\
\left(\sigma_{\mathrm{s}}\right)\end{array}$ & $\begin{array}{c}\text { Constitutive } \\
\text { relation }\end{array}$ & $\begin{array}{c}\text { Density } \\
(\rho)\end{array}$ \\
\hline $210 \mathrm{GPa}$ & 0.3 & $290 \mathrm{MPa}$ & $\sigma=395 \times \varepsilon^{0.05}$ & $7800 \mathrm{~kg} \mathrm{~m}^{-3}$ \\
\hline
\end{tabular}

rollers. During the entire process of line rolling forming, the springback produced by unloading exists, so it is necessary to predict mechanical springback. In the simulation, the relative gap and contact pressure between the rollers and the plate is used to determine whether they are in contact or separated. After the static load is removed, it is assumed that the roller is separated from the pressed plate, and the resilience calculation is carried out.

The established finite element model is presented in Fig. 2. The directions of the short and long edges of the plate are defined as $x$ - and $y$-axes respectively, and its thickness direction corresponds to the $z$-axis. In addition, the origin of the axes coordinates is on the center of the pressed plate. Within the contact region of the upper and lower rollers, grid elements are appropriately refined, together with transitional grid generation out of the contact region.

Geometric and material parameters of the pressed plate adopted for the associated calculation model are presented in Table 1. A power hardened and incompressible material is adopted, and the stress-strain curve is derived from previous experimental results (Wu, 2014).

The geometrical shapes and dimensions of the rollers are shown in Fig. 3. The rollers apply a press amount rolling load equal to 0.2 times the plate thickness onto the pressed plate.

To verify the applicability of this method, a threedimensional elastoplastic finite element calculation is performed for comparison with the experimental results in Shaohui's results (Shaohui et al., 2012), wherein the abovementioned similar computational features were applied. The comparison results are presented in Table 2, where $2 \mathrm{a}$ is the distance between the support rolls, $d_{Z}$ is the forming depth, $\mathrm{Rb}$ is the radius of curvature of the workpiece boundary, $(\mathrm{Rb})_{\text {EXP }}$ refers to the experimental results and $(\mathrm{Rb})_{\mathrm{CAL}}$ refers to the FEM results. The excellent correlation between the numerical and experimental results is revealed in Table 2, demonstrating the feasibility of the three-dimensional elastoplastic finite element method.

In accordance with the objective of the simplified deformation simulation method mentioned above, the aim is to accurately simulate the deformation shapes of the pressed plate after rolling loading. In other words, determining the transient variation in mechanical quantities in the process of rolling loading as well as the calculation of other nongeometrical mechanical quantities are not the main concerns. From the perspective of geometrical relationships, deforma- 
Table 2. Comparison of experimental and FEM results (Yoon et al., 2003).

\begin{tabular}{lccccc}
\hline \multirow{2}{*}{ No. } & $2 \mathrm{a}$ & $d_{Z}$ & \multicolumn{3}{c}{$\mathrm{Rb}(\mathrm{mm})$} \\
\cline { 4 - 6 } & $(\mathrm{mm})$ & $(\mathrm{mm})$ & $(\mathrm{Rb})_{\mathrm{EXP}}$ & $(\mathrm{Rb})_{\mathrm{CAL}}$ & $(\mathrm{Rb})_{\mathrm{EXP}} /(\mathrm{Rb})_{\mathrm{CAL}}$ \\
\hline 1 & 40 & 1.2 & 142.4 & 149.3 & 0.95 \\
2 & 45 & 1.2 & 191.8 & 198.5 & 0.96 \\
3 & 40 & 1.4 & 125.4 & 134.2 & 0.93 \\
4 & 45 & 1.4 & 167.0 & 172.7 & 0.97 \\
\hline
\end{tabular}

tion in its ultimate state after loading and unloading shows a one-to-one correspondence with strain at that time. Therefore, the strain distribution for the entire plate should be found, which is directly associated with the determination of plate shape.

Rolling forming is a large deformation process; the relationships between the corresponding deformation and the strain can be expressed as follows.

$$
\left\{\begin{array}{l}
\varepsilon_{x}=\frac{\partial u}{\partial x}+\frac{1}{2}\left(\frac{\partial w}{\partial x}\right)^{2}-z \frac{\partial^{2} w}{\partial x^{2}} \\
\varepsilon_{y}=\frac{\partial v}{\partial x}+\frac{1}{2}\left(\frac{\partial w}{\partial y}\right)^{2}-z \frac{\partial^{2} w}{\partial y^{2}} \\
\gamma_{x y}=\frac{\partial u}{\partial y}+\frac{\partial v}{\partial x}+\frac{\partial w}{\partial x} \frac{\partial w}{\partial y}-2 z \frac{\partial^{2} w}{\partial x \partial y}
\end{array}\right.
$$

where $\varepsilon$ and $\gamma$ as well as their subscripts refer to the axial strain and shearing strain in one direction, respectively. Considering the thinness characteristics of the shell plate, $\varepsilon_{z}=\gamma_{y z}=\gamma_{z x}=0$. As described above, when rolling depression loading and unloading are completed, its strain element should correspond to the strain, just as the deformation given in Eq. (1) corresponds to the ultimate residual deformation of the pressed plate. For the convenience of describing the strain, the strain components obtained through calculations are transformed into a principal strain expression. Hence, based on the above strain, the corresponding principal strain and direction can be expressed in the following equation set.

$$
\left\{\begin{array}{l}
\varepsilon_{1}=\frac{\varepsilon_{x}+\varepsilon_{y}}{2}+\frac{1}{2} \sqrt{\left(\varepsilon_{x}-\varepsilon_{y}\right)^{2}+\gamma_{x y}^{2}} \\
\varepsilon_{2}=\frac{\varepsilon_{x}+\varepsilon_{y}}{2}-\frac{1}{2} \sqrt{\left(\varepsilon_{x}-\varepsilon_{y}\right)^{2}+\gamma_{x y}^{2}} \\
\alpha=-\frac{1}{2} \arctan \left(\frac{\gamma_{x y}}{\varepsilon_{x}-\varepsilon_{y}}\right)
\end{array}\right.
$$

where $\varepsilon_{1}$ and $\varepsilon_{2}$ are the first and the second principal strains respectively; $\alpha$ refers to the direction angle of the principal strain. Considering the relationship between pressed plate forming and the in-plane/bending strain, the distributions of principal strains are decomposed into in-plane and bending strains. Then, $\varepsilon_{i}^{m}$ and $\varepsilon_{i}^{b}(\mathrm{i}=1,2)$ are respectively used to represent the in-plane and bending strain components of the principal strain, which can be obtained based on the following equation set.

$$
\left\{\begin{array}{l}
\varepsilon_{i}^{m}=\frac{1}{h} \int_{-h / 2}^{h / 2} \varepsilon_{i} \mathrm{~d} z \\
\varepsilon_{i}^{b}=\frac{2}{h^{2}} \int_{-h / 2}^{h / 2} z\left(\varepsilon_{i}-\varepsilon_{i}^{m}\right) \mathrm{d} z
\end{array}\right.
$$

where $h$ stands for the plate thickness. The in-plane and bending strain direction for principal strain is still denoted by $\alpha$ from Eq. (2).

The three-dimensional elastoplastic finite element calculation results are shown in Fig. 4. Specifically, the overall deformation $W$ and the curvature $K$ as well as their subscripts denoting direction are separately represented by results of the sections from a-a to $\mathrm{f}-\mathrm{f}$ along the directions of plate $x$ and $y$. According to Fig. 4a-d, the local line rolling loading is able to generate deformations and curvatures in two directions simultaneously, and the curvature in the $x$ direction is small; Based on the existing press amount, the maximum deflection ratio and curvature ratio on directions of $y$ and $x$ are approximately 5 and 100. Further, it can be seen from Fig. 4a and b that the regions where deformation in the $y$ direction takes place are basically centralized between two contacts points of the lower concave wheel, that is the rolling depression part. From the results for sections a-a to $d-d$ of the pressed plate perpendicular to the $y$ direction of the rolling line, it is clear that the $\mathrm{b}-\mathrm{b}$ and $\mathrm{c}-\mathrm{c}$ sections near the middle part of the pressed plate have fundamentally similar deformation sizes and curvatures. There is a greater difference however between the $\mathrm{a}-\mathrm{a}$ and $\mathrm{d}-\mathrm{d}$ sections. In the existing condition of local line rolling loading, except smaller scopes on both loading terminals of the pressed plate, its deformation and curvature can stay stable for a certain duration. Thus, that with the increase in plate length can be inferred, such a feature will become increasingly prominent.

If the strain of the pressed plate is transformed according to Eqs. (2) and (3), the corresponding results expressed in principal in-plane and bending strains can be seen in Fig. 5a and $b$. Figure $5 \mathrm{c}$ shows the distribution of maximum principal strain. The orthogonal line segments of one point stand for the first and the second principal strain; the length of an arrow signifies the size of such a strain, and its direction denotes the direction of strain. Two arrows extending outward indicate a positive value; else, the value is negative. In Fig. 5a and $b$, changes in the principal in-plane and bending strains along the $x$ and $y$ coordinates of the pressed plate are shown. For the in-plane strain, two outward arrows refer to tension strain, while two inward arrows refer to compression strain. In contrast, with regard to the bending strain, the direction of arrows indicates the bending direction of the plate; two outward arrows refer to upward bending, while two inward arrows refer to downward bending.

As seen in Fig. 5c, all strains are concentrated within the region near the rolling line, approximately in the range of the 
(a)



(c)

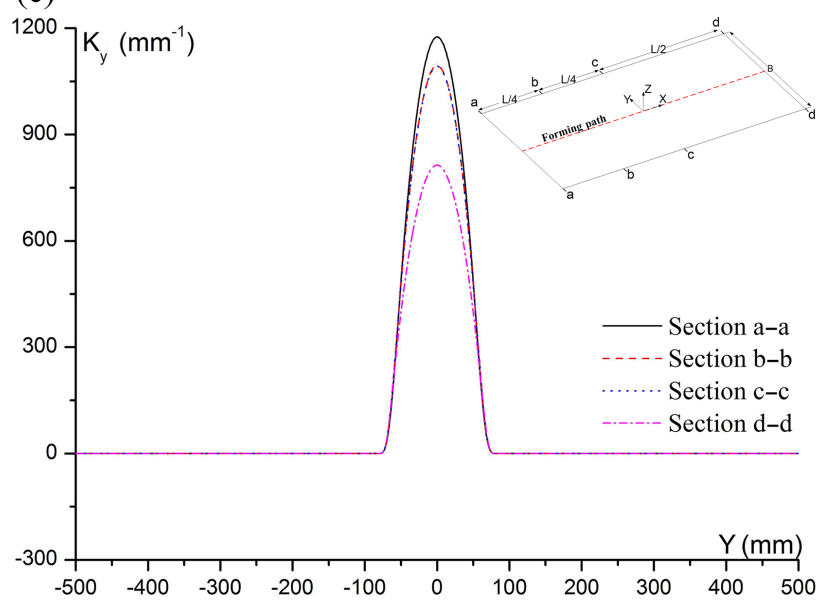

(b)

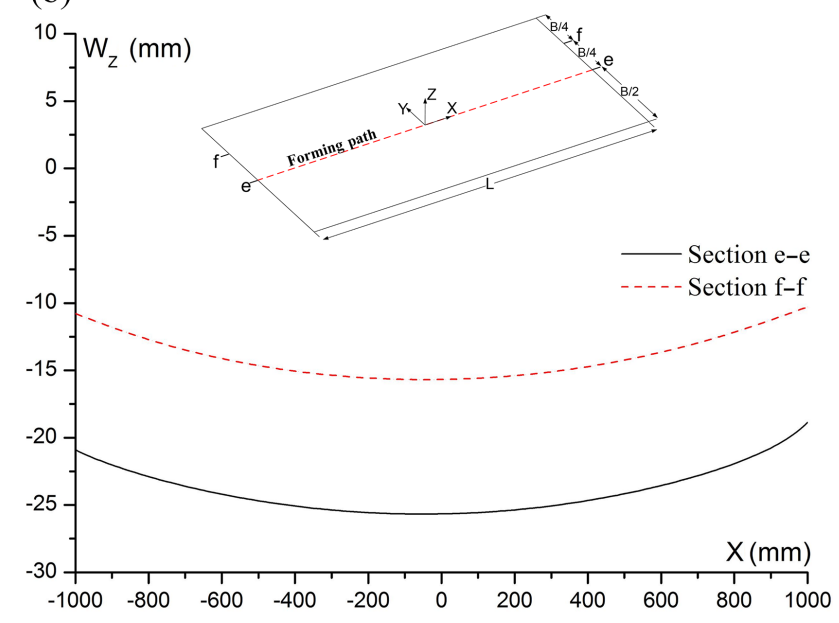

(d)

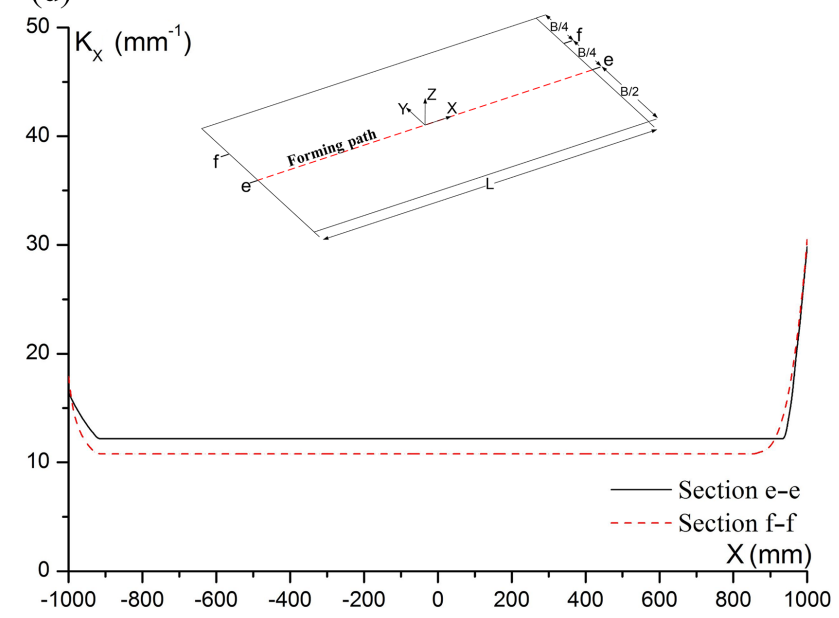

Figure 4. Results of deformation and curvature $(\delta=0.2 \mathrm{~h})$. (a) Deformation in $y$ direction. (b) Deformation in $x$ direction. (c) Curvature in $x$ direction. (d) Curvature in $y$ direction.

lower roller wheel width, which is approximately $180 \mathrm{~mm}$. Deviating from such a region, the strain distribution is almost zero. Furthermore, inside this region, they mainly are together near the center line of rolling depression; in comparison with the midpoint value, that of strain near the center line of rolling depression decreases and rapidly approaches zero. In addition, not only are the maximum in-plane and bending strains of the first principal strain larger than those of the second principal strain, but its direction is perpendicular to the direction of the loading line. In addition to the rising and descending of the starting and ending points of the strain of the rolling loading path owing to the edge effect, the distribution of strains along the rolling loading path almost remains unchanged. Both in-plane and bending strains have the above features. As for the in-plane strain, they are mainly tensile strains vertical to the loading path, while elements of bending strain are upward bending strains vertical to such paths. Their maximum values are 0.003 and 0.024 , respec- tively. Clearly, the bending strain is one order of magnitude greater than the in-plane strain, indicating that the bending strain is a major component of deformation depending on the extent of pressing.

On the premise that a press amount of 0.2 times the plate thickness remains for rolling loading, the in-plane and bending strains are determined on different unloading positions of the pressed plate. They are from one end of this plate to $1 / 4$, $1 / 2$, and $3 / 4$ of its length. Figure 6 shows the variation in distribution of the in-plane and bending strains on the center line of the pressed plate. As seen in the figure, irrespective of the location of the termination point of the loading path, the in-plane and bending strains are both at a steady stage throughout the rolling path, despite that they go up and down within a very small scope from the initiating terminal and of loading termination (unloading). Their values are certain and fundamentally coincide with each other. 
(a)

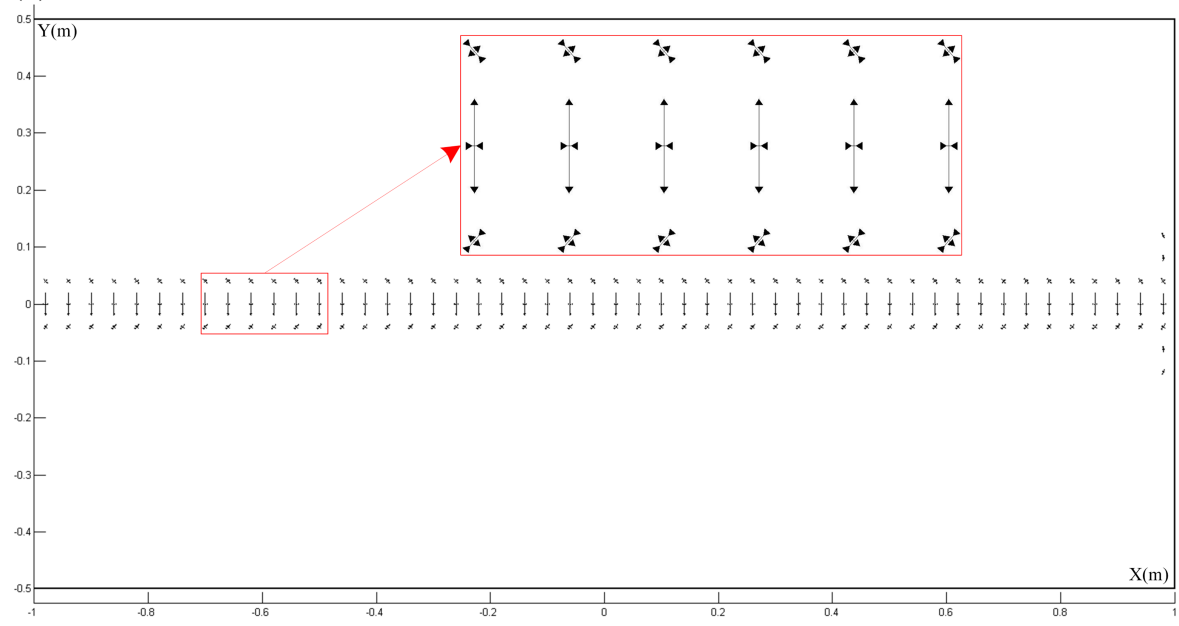

(b)

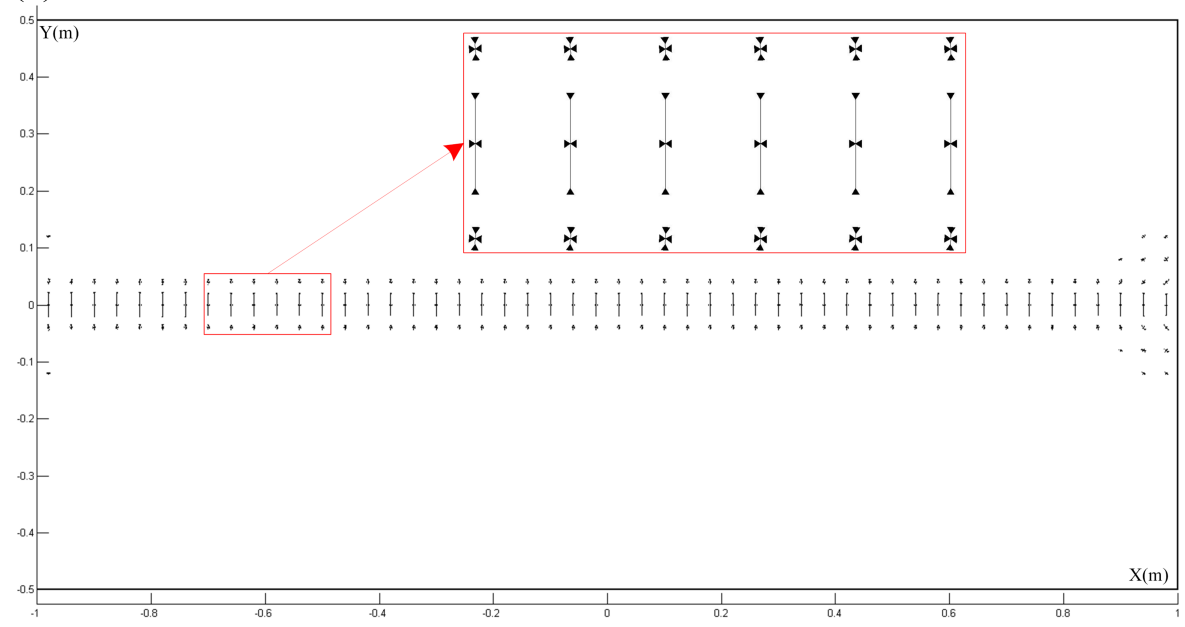

(c)

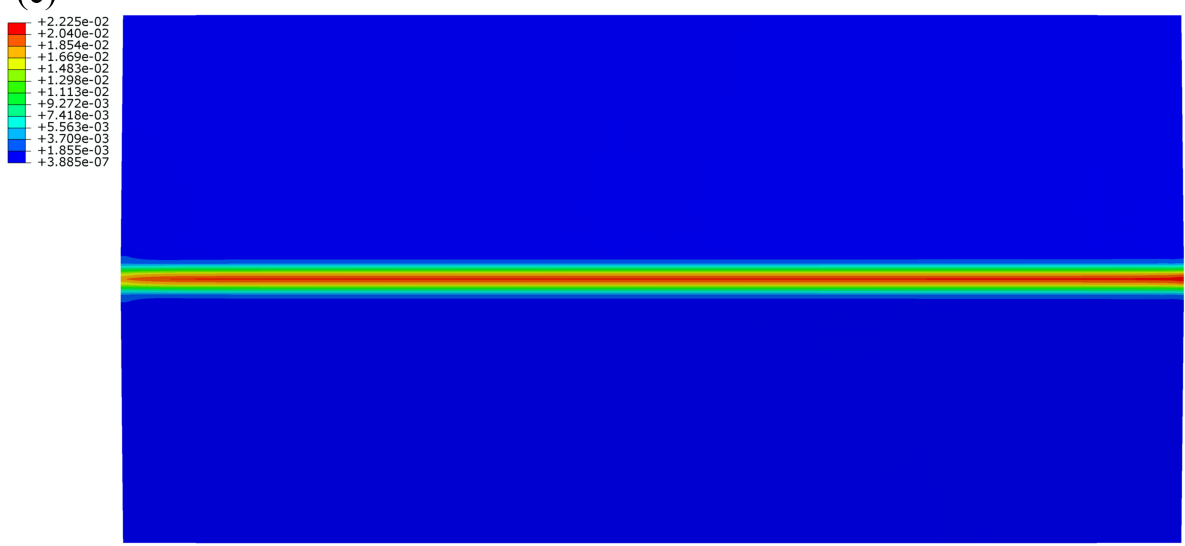

Figure 5. Results of strain distributions. (a) Distribution of in-plane strain (maximum value $=0.003$ ). (b) Distribution of bending strain (maximum value $=0.024)$. (c) Distribution of maximum principal strain.

Figure 7 shows the calculation results obtained by changing the initial press amount. According to it, the maximum in-plane principal strain and the maximum bending princi- pal strain both increase with the press amount. However, the change laws of the corresponding strain values remain the same. In other words, within a very small area of loading 
(a)

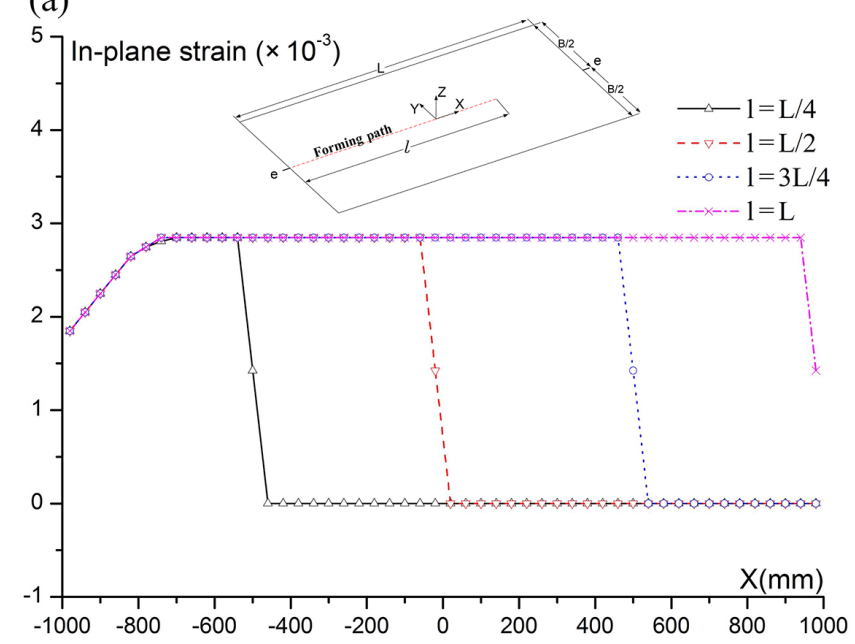

(b)

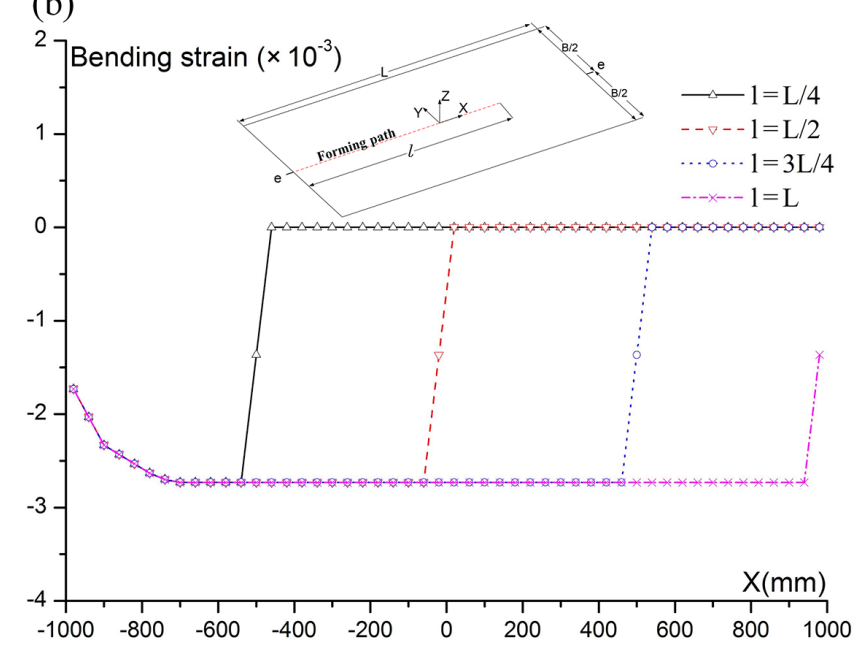

Figure 6. Relationship between length of loading path and midline strain variation of the rolling line of pressed plate. (a) In-plane strain distribution. (b) Bending strain distribution.

(a)

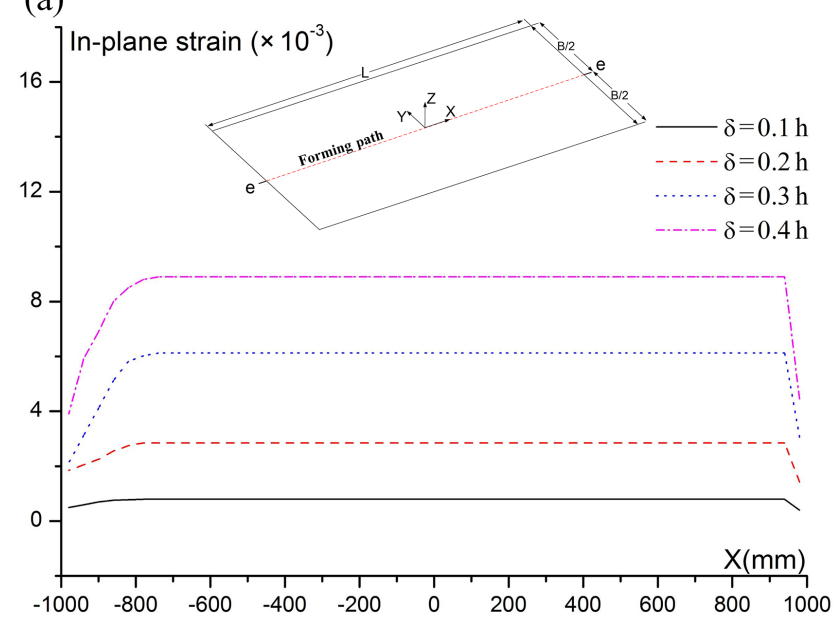

(b)

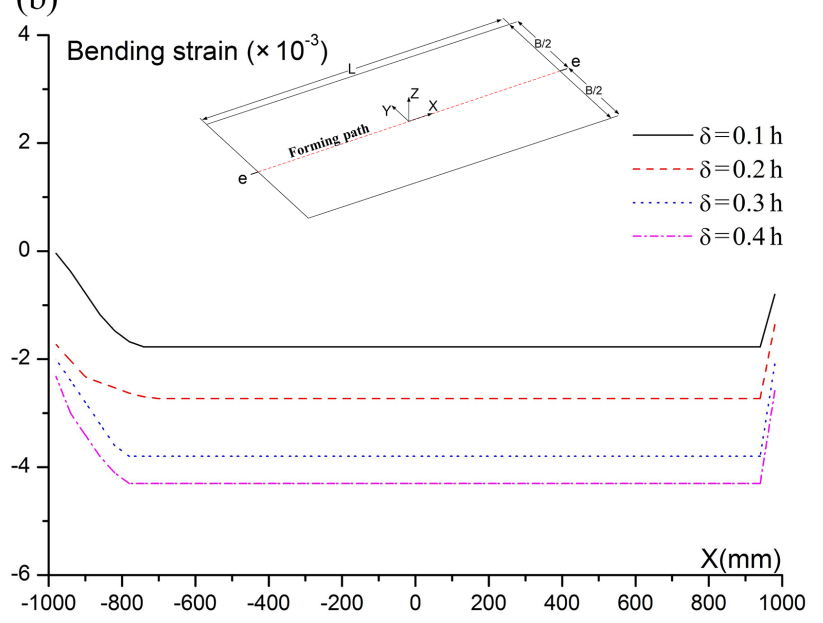

Figure 7. Relationship between press amount and midline strain variation of the rolling line of pressed plate. (a) In-plane strain distribution. (b) Bending strain distribution.

away from the initiating terminal and within a very small area of loading termination, the in-plane and bending strains both maintain definite values throughout the rolling path. As the press amount increases, the lengths of such two variation areas may increase slightly. However, they are still very short when compared to the entire rolling line.

Figure 8 shows the calculation results of the diverse thicknesses of the plate. Clearly, as the thickness of the plate rises, the distributions of the relevant strains also change. In addition, the maximum in-plane principal strain decreases while the maximum bending principal strain increases. Meanwhile, the lengths of the strain variation regions at the initiation and termination of loading increase slightly despite the corresponding variations being very small. As for in-plane and bending strains, they remain stable on a majority of the rolling loading paths.

According to results shown in Figs. 6-8, as long as the loading length of the plate is not excessively short, changes in the loading path length, press amount, and plate thickness have no significant influence on the lengths of the strain increase and decrease segments at the initiation and termination of loading. In in most cases, in practical manufacturing processes, the loading path can be very long. Therefore, it can be considered that in-plane and bending strains basically remain invariant for most of the entire rolling loading path. 
(a)

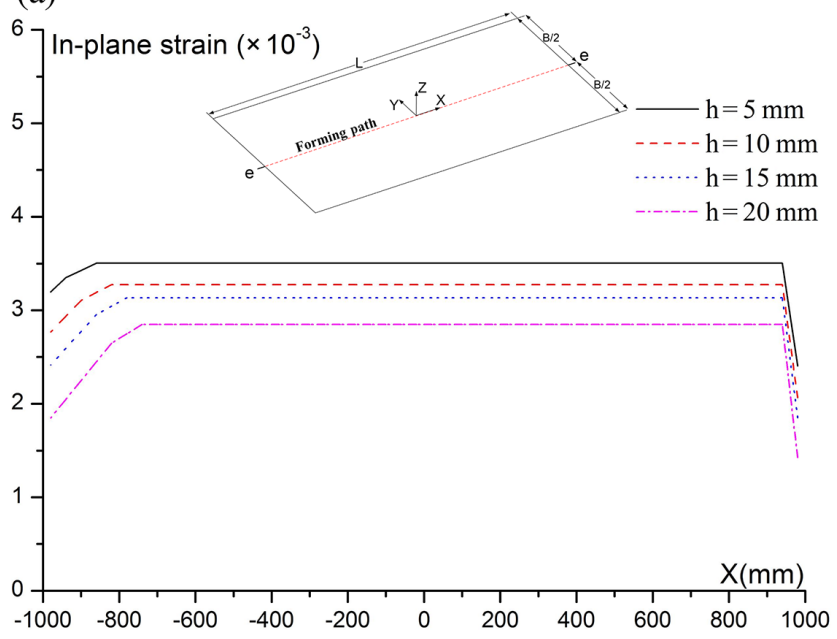

(b)

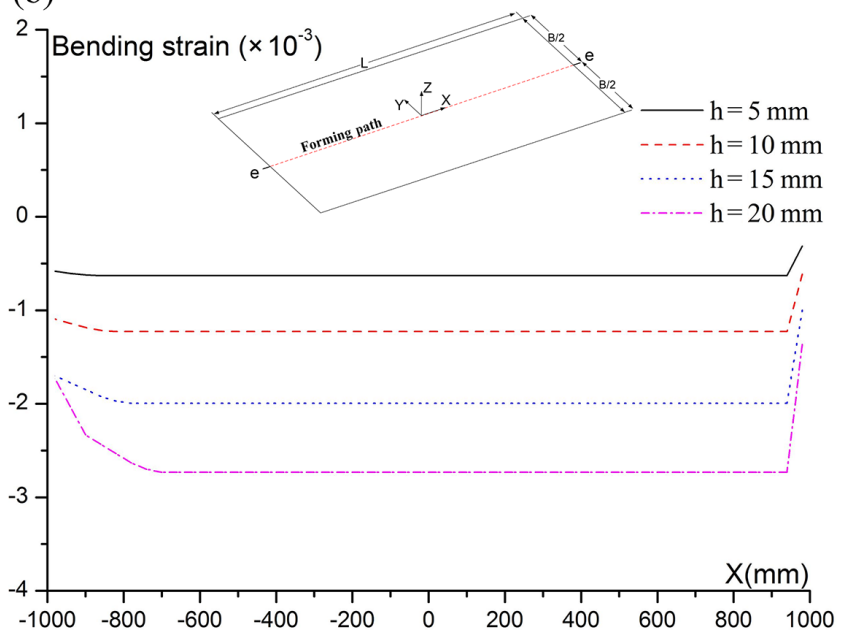

Figure 8. Relationship between plate thickness and midline strain variation of the rolling line of pressed plate. (a) In-plane strain distribution. (b) Bending strain distribution.

\section{Simplified deformation simulation method}

The above three-dimensional elastoplastic finite element calculation results demonstrate the basic strain distribution characteristics of local line rolling. First, the strain is mainly concentrated near the center line of rolling. Second, within a very short distance from loading initiation and termination, the strain increases or decreases; it remains at a constant value when it is not affected by the length of loading path, press amount, and the thickness of the plate. In addition, in-plane and bending strains follow the same distribution law.

Depending on the above characteristics, the method mentioned below can be adopted to replace the three-dimensional elastoplastic finite element for performing the relevant calculations. The calculation based on the three-dimensional elastoplastic finite element is performed first for single shortrange rolling line loading under diverse press amounts. Here, the short range refers to the minimum distance to reach a stable strain stage described above. According to the previous results, it is known that the loading path is generally able to attain a stable area of strain through approximately 10 times plate thickness from the beginning. In fact, during such a calculation, the corresponding relationship between press amount and strain is established simultaneously. That is, the calculation depends on the relationship between the pressed plate deformation and some process parameters. Subsequently, the variations in strain at the beginning and end of the loading line should be neglected, so as to extract all strain components of a stable strain region, which are determined by the three-dimensional elastoplastic finite element calculation. Furthermore, these components approximately serve as strain values on the complete loading path. These strains are applied onto the pressed plate by means of a linear elastic calculation based on the mode of initial strain. There-

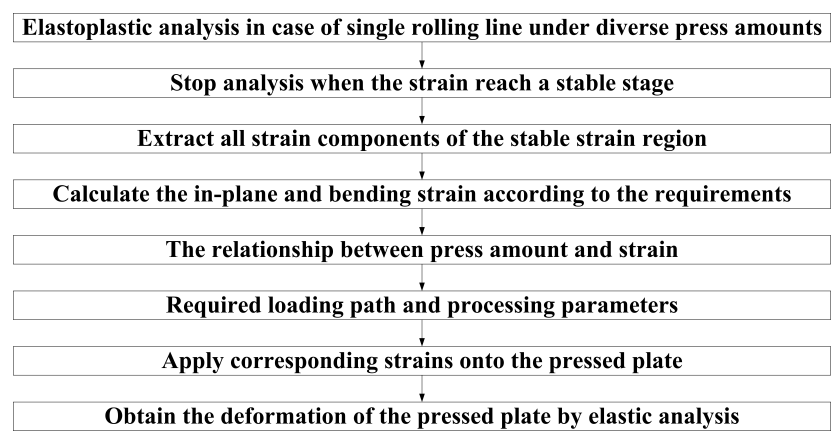

Figure 9. Simplified deformation simulation method.

fore, deformations of the pressed plate under different press amounts or various lengths/directions of loading paths can be obtained. The advantage of such a computational method is the substantial reduction in the three-dimensional elastoplastic finite element calculations. The extraction of effective strains, centralized within a narrow area of the pressed plate requires little workload. While the secondary calculation is an elastic calculation that applies a strain with low approximation, the original features are essentially maintained. The computational method described above is referred to as the simplified deformation simulation method, and the corresponding computational flow is summarized in Fig. 9.

Depending on the simplified deformation computation method, the large deformation elasto-plastic finite element method is adopted to establish the correspondence between strain distribution and processing parameters. On this basis, calculation of elasticity can be carried out to acquire the ultimate deformation result. Compared to the large deformation elasto-plastic finite element method, the simplified deformation computation method omits iterative computations 
(a)

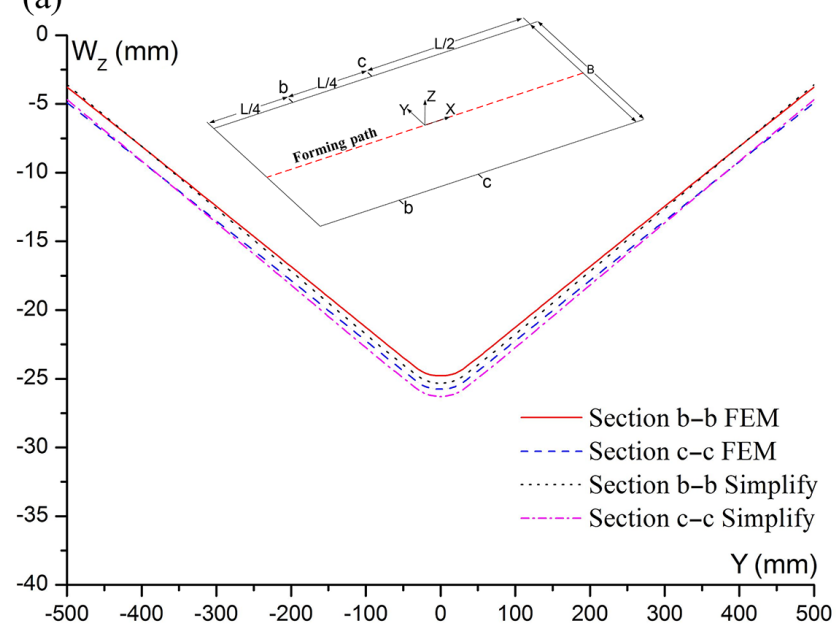

(b)



Figure 10. Comparison of deformation between elastoplastic FEM and simplified deformation simulation method. (a) Results from lateral sections. (b) Results from longitudinal sections.

required by the nonlinear finite element method during forming and springback simulations. Instead, it directly exerts initial strain distribution on the plate model to obtain the final deformation outcome by means of large deformation elasticity analysis. Specifically, the large deformation elasto-plastic finite element method can be used to follow variations in the stress strain and displacement field for the entire forming process; in contrast, the simplified deformation computation method focuses on deformation results after forming. Evidently, such a difference is the fundamental reason why the former requires a long computing time and a large amount of computation, while the latter requires a short computing time and a small amount of computation.

\subsection{Comparison between elastoplastic FEM and simplified method}

In order to test the computational accuracy and efficiency of the simplified deformation simulation method, based on the premise that calculation procedures of this method presented in Fig. 8 are followed, the comparison calculation is carried out under conditions of pressed plate and rolling loading; these conditions are identical to those adopted by the three-dimensional elastoplastic finite element calculation in the above section. The computational grid of the simplified deformation simulation method is not required to be consistent with that of the three-dimensional elastoplastic finite element calculation. As the simplified deformation simulation method falls into the elastic calculation category, the requirement for the element grid is rather low. For the convenience of mesh generation and adopting an identical element grid calculation model to determine multiple rolling loading paths, homogeneous element grids that are divided in line with appropriate density can be selected to perform calculation based on the simplified deformation simulation method. Thus, it is conceivable that grids cannot be divided in accordance with Fig. 2 under the condition of multiple rolling loading paths as far as the three-dimensional elastoplastic finite element calculation is concerned. Consequently, full-plate grid subdivision must be conducted for the pressed plate with the aim of adapting rolling loading paths to different directions and positions.

First, three-dimensional elastoplastic finite element calculation is carried out. The calculation ends when the length of the corresponding loading path is approximately 10 times the plate thickness. After all strain components are extracted near the single rolling line, they are applied to every element of an identical loading path of the pressed plate as initial strains to perform elasticity calculations. In this way, the deformation of such a pressed plate can be acquired. The calculation results are compared with deformations of the vertical and horizontal cross sections, which are obtained by the three-dimensional elastoplastic finite element calculation, and with the deformations of sections $\mathrm{b}-\mathrm{b}, \mathrm{c}-\mathrm{c}, \mathrm{e}-\mathrm{e}$ and $\mathrm{f}-\mathrm{f}$ obtained by the three-dimensional elastoplastic finite element calculation. The related locations of sections for such comparisons are shown in Fig. 10. It can be seen that along the $x$ - and $y$-axes of the pressed plate, the deformation results achieved by the simplified deformation simulation method perfectly coincide with those obtained using the three-dimensional elastoplastic finite element calculation.

The calculation time comparisons between the two methods are presented in Table 3; it is clear that the calculation time required by the simplified deformation simulation method is $1 / 100$ of that required by the three-dimensional elastoplastic finite element method. The full-plate uniform rectangular grid partition with 200 and 100 elements on the $x$ and $y$-axes, respectively, is considered in the simplified de- 
(a)

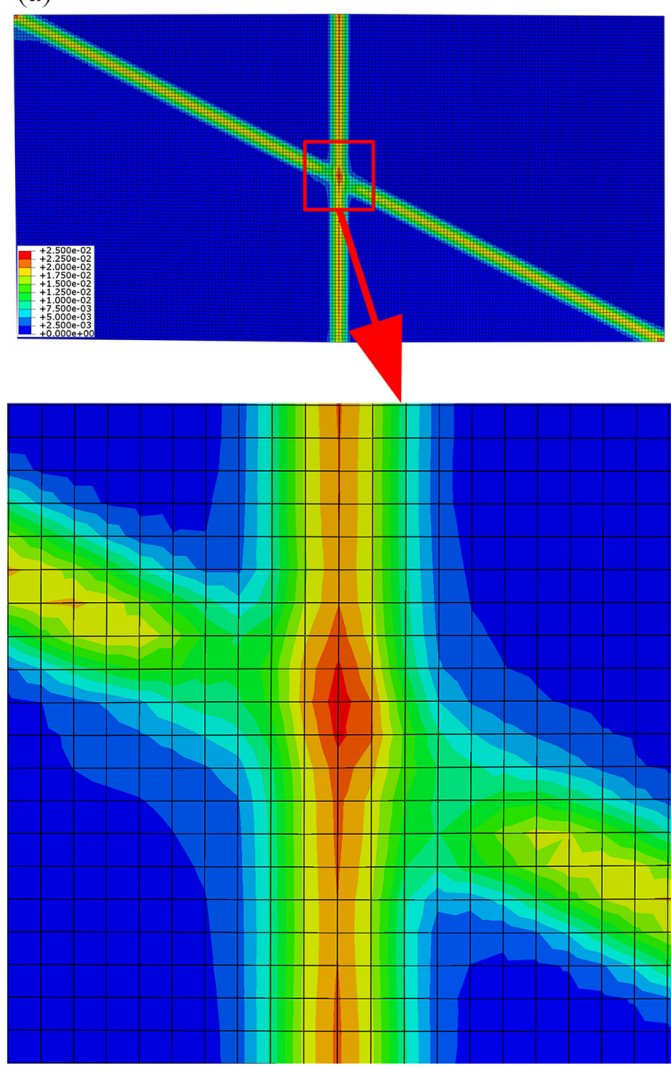

(b)



Figure 11. Maximum principal strain distribution of different loading sequences. (a) Loading scheme 1. (b) Loading scheme 2.

Table 3. Comparison for elastoplastic FEM and simplified deformation simulation method.

\begin{tabular}{lcrrr}
\hline Method & $\begin{array}{c}\text { Number of } \\
\text { elements }\end{array}$ & $\begin{array}{r}\text { Number of } \\
\text { nodes }\end{array}$ & $\begin{array}{r}\text { Incremental } \\
\text { steps }\end{array}$ & Time \\
\hline Elastoplastic FEM & 29200 & 37067 & 2001 & $960 \mathrm{~min}$ \\
Simplified method & 80000 & 101505 & 1 & $10 \mathrm{~min}$ \\
\hline
\end{tabular}

formation simulation method. In other words, the numbers of elements and nodes are both greater than those of the three-dimensional elastoplastic finite element method. Despite such facts, the calculation time taken is far less than that required by the three-dimensional elastoplastic finite element method.

\subsection{Application of the simplified deformation simulation method}

In reality, local line rolling forming is the process of multichannel rolling processing in diverse directions. Without loss of generality, two rolling loading paths in diverse directions are selected for this study to perform the three-dimensional elastoplastic finite element method; moreover, the simplified deformation simulation method is also adopted to carry out comparison calculations, so as to validate the applicability of such a simplified deformation simulation method under the condition of multiple loading lines. According to the preceding calculation results, deformation or plastic zone is focused within a narrow band under the action of rolling loading. Approximately, it lies between the widths of lower rollers. Therefore, if the width of the rollers do not overlap with each other for twice rolling, the loading sequence may not have an enormous impact on the deformation results of the pressed plate. However, in order to investigate effects of the loading sequence, calculations are also conducted for various loading sequences. By taking the existing calculation results given in the previous section, direction $y$ from the long edge midpoint of the pressed plate and one of its diagonals are selected as the two loading paths. They have a point of intersection; the associated calculation conditions are the same as those given in Tables 1 and 3. The press amount of roller is taken to be 0.2 times that of the plate thickness. Here, the loading along the $y$ direction from the long edge midpoint of the pressed plate is referred to as Loading Scheme 1; while, that along the diagonal is known as Loading Scheme 2. Figure 11 shows the dependency of strain distributions on loading sequence. According to this figure, the two loading schemes only show evident differences at the intersection of loading lines as far as plastic strain distributions are concerned. 
(a)

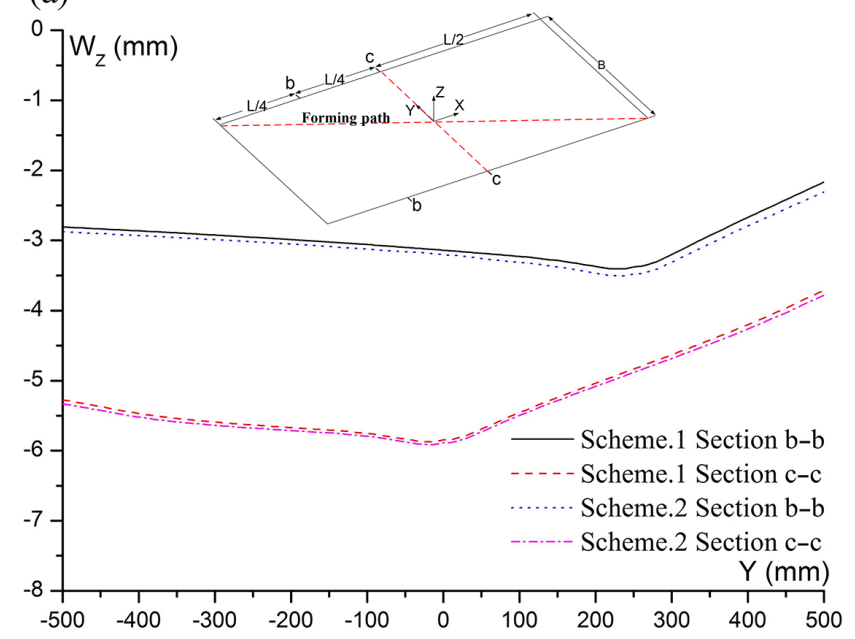

(b)

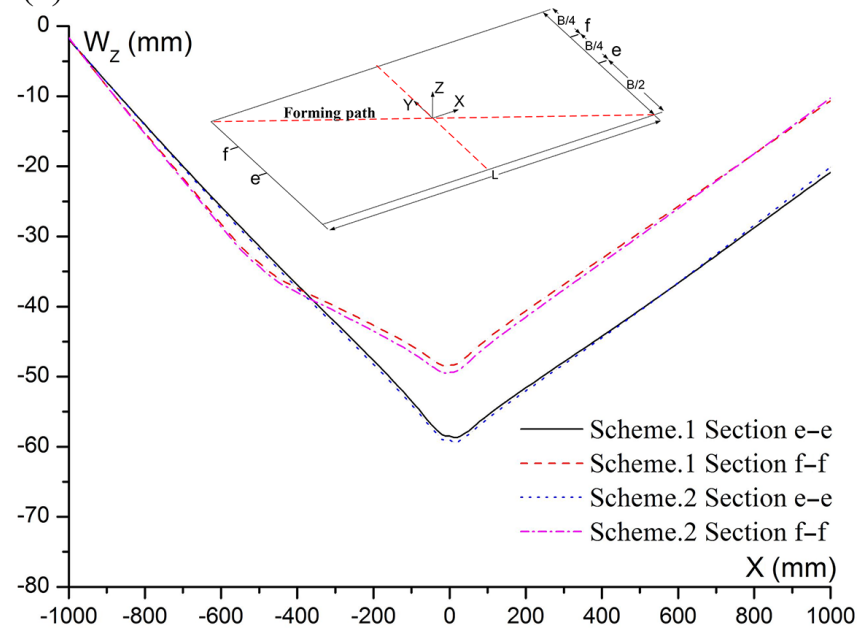

Figure 12. Results of deformation of different loading sequences. (a) Results from lateral sections. (b) Results from longitudinal sections.

(a)

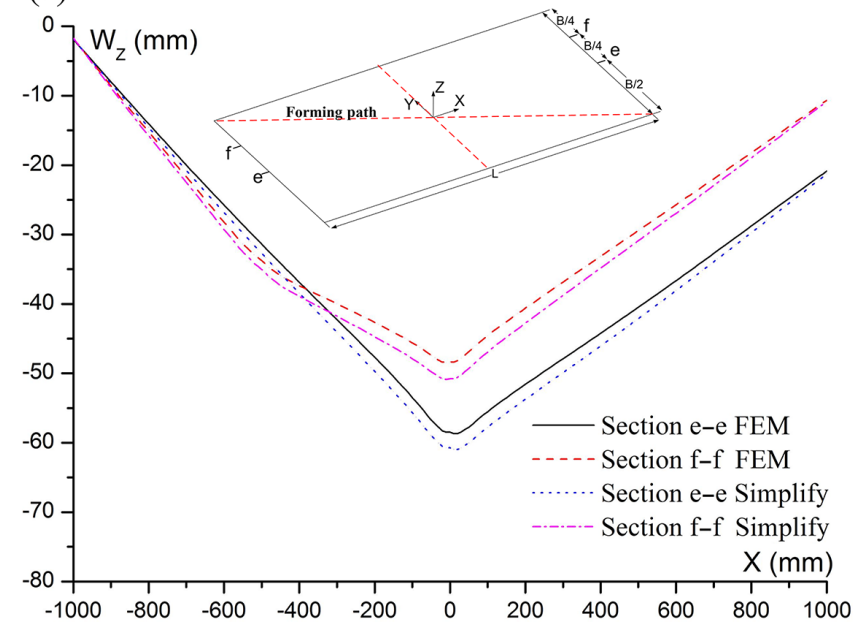

(b)

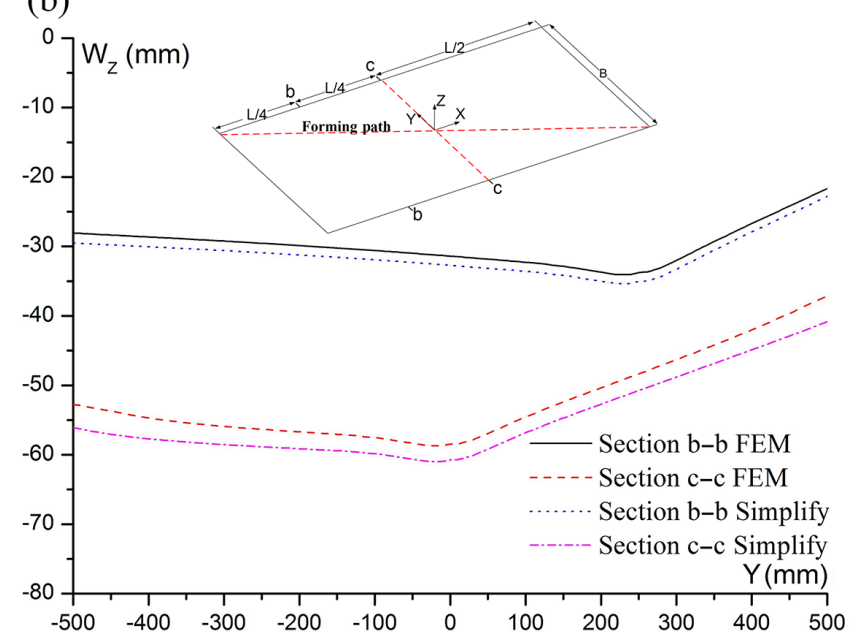

Figure 13. Comparison of deformation between elastoplastic FEM and simplified deformation simulation method (two lines). (a) Results from longitudinal sections. (b) Results from lateral sections.

In addition, the corresponding variation range is very small and its size is only approximately $100 \times 100 \mathrm{~mm}$ (element size $=10 \times 10 \mathrm{~mm}$ ). From the perspective of area, it occupies approximately $1 / 200$ of the total area of the plate. In terms of length, it is approximately $1 / 20$ of the shorter loading line. Figure 12 shows the deformation comparison results for such two schemes of loading sequence. They indicate that almost no differences lie in their deformations. With the increase in press amount, such an involved area may expand appropriately. However, its suffering area is mainly concentrated within a very small area around the intersection. Clearly, such a characteristic has no dramatic changes. In other words, such a variation has no significant influence on the entire deformation of the pressed plate.
The calculation results of the three-dimensional elastoplastic finite element method and simplified deformation simulation method are shown in Fig. 13. The calculation results indicate that for two loading lines, a preferable coincidence exists between the calculation results of the simplified deformation simulation method and those of the threedimensional elastoplastic finite element method in terms of deformations. Deformation differences caused by the compliance of plastic deformation sequences near the intersection of loading lines can be neglected. Therefore, the simplified deformation simulation method is applicable to calculations of multiple loading lines. Concerning the calculation time comparisons of two loading lines, please see Table 4. According to this table, it is clear that the simplified deformation simulation method is more computational efficient. 
(a)

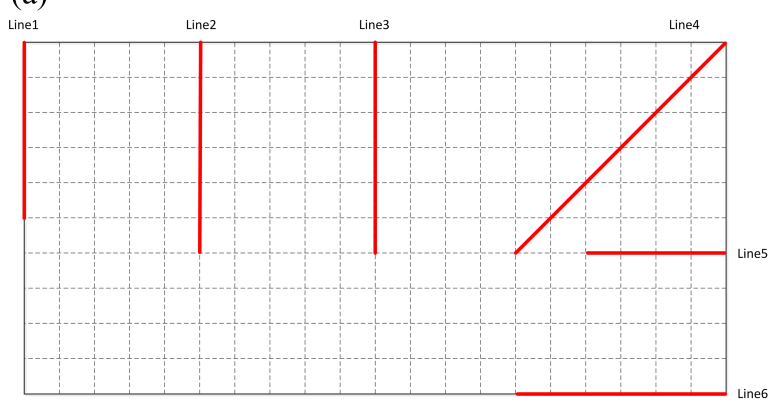

(b)

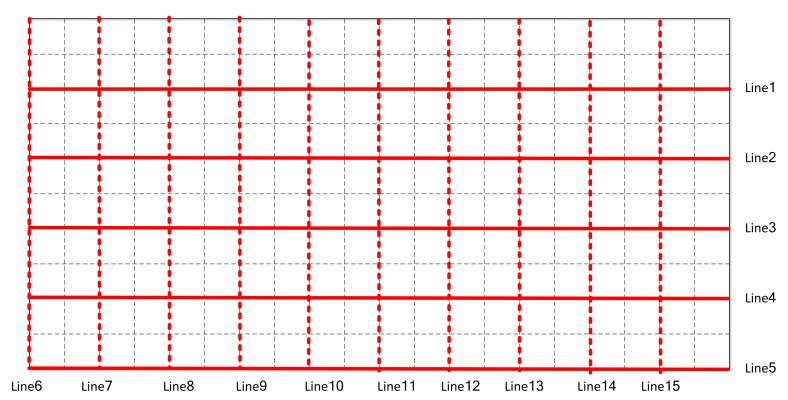

Figure 14. Schematic diagram for two different rolling line loading paths. (a) Path 1. (b) Path 2.

Table 4. Comparison for elastoplastic FEM and simplified deformation simulation method (two lines).

\begin{tabular}{lcrrr}
\hline Method & $\begin{array}{c}\text { Number of } \\
\text { elements }\end{array}$ & $\begin{array}{r}\text { Number of } \\
\text { nodes }\end{array}$ & $\begin{array}{r}\text { Incremental } \\
\text { steps }\end{array}$ & Time \\
\hline Elastoplastic FEM & 29200 & 37067 & 3400 & $4000 \mathrm{~min}$ \\
Simplified method & 80000 & 101505 & 1 & $15 \mathrm{~min}$ \\
\hline
\end{tabular}

In accordance with the above results, after the practical plate forming loading scheme has been acquired from key points (i) and (ii) in the actual calculation, simplified deformation simulation computing can be adopted during verification computation according to all loading paths and the relevant loading processes so as to complete strain application on the corresponding elements at a time, provided that the widths of loading paths do not overlap with each other. Then, an elasticity calculation is able to achieve deformation of the pressed plate. As an application of the simplified deformation simulation method, the possibility of adopting this method to realize complex curvature shape calculations through multiple loading paths is explored.

In this study, the formation of a sailed plate and a saddle plate is taken as an example to define process parameters related to such forming. The loading path obtained by the above method is shown in Fig. 1, which can be divided into three steps.

- In Step 1, the large deformation elastic finite element method is used to obtain the required strain field distribution according to the target shape desired. As the length and width of planking are both larger than its thickness, the strain distribution is decomposed into the in-plane strain and the bending strain.

- In Step 2, the processing path is determined in accordance with distributions of in-plane and bending strains. In-plane and bending strain distributions can be utilized to determine the processing path of local line rolling because local line rolling forming mainly leads to bending and in-plane tensile strains perpendicular to such a path.
In addition, the workpiece surface for loading can be also confirmed based on bending strain symbols here.

- In Step 3, the processing parameters are determined according to the processing path. As both the shape and dimension of the roller remain unchanged in this study, this step principally aims at making the corresponding loading force clear.

Owing to symmetry, only $1 / 4$ of the pressed plate is shown in this figure. Concerning the loading Path 1 for the pressed plate, 20 loading lines are chosen in total, and all loading lines loading on an identical surface of the pressed plate. For loading Path 2, 28 loading lines are adopted. While 19 of those loading lines act on one surface of the pressed plate (denoted by the dotted lines in Fig. 14b), another 9 act on the other surface of the pressed plate (signified by the full lines in Fig. 14b).

After determination of the loading paths, the next step is to determine the press amount. It is done as follows:

- Step 1: in terms of all the determinated loading paths, calculating the average value of in-plane principal strain or bending principle strain along loading path and calculating integrals to get total in-plane or bending strain.

- Step 2: using elastoplastic finite element method to simulate the local line rolling forming to get the strain distribution on pressed plate. Transferring strain into the forms of in-plane strain and bending strain by Eqs. (2) and (3) and calculating integrals along plastic zone to get total in-plane strain and bending total strain.

- Step 3: according to Step 2 calculating a series of values under conditions of different press amounts, getting the database of relationships bewteen press amount and total in-plane strain or total bending strain.

- Step 4: matching the total in-plane strain or total bending strain with data in database of Step 3, selecting the press amount which total strain is closest to the total strain of Step 1. 
(a)

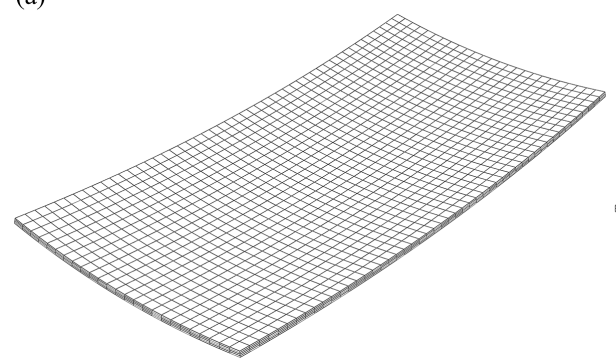

(b)

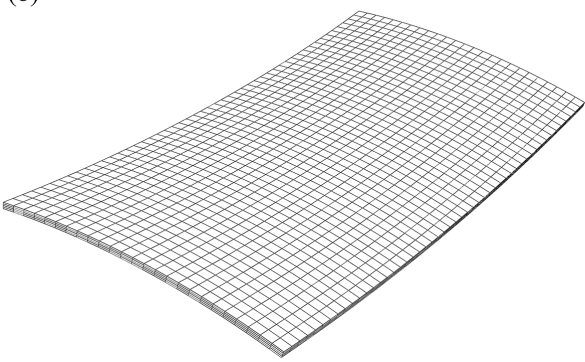

Figure 15. Deformation results for diverse loading paths based on the simplified deformation simulation method. (a) Result for Path 1 of pressed plate. (b) Result for Path 2 of pressed plate.

(a)

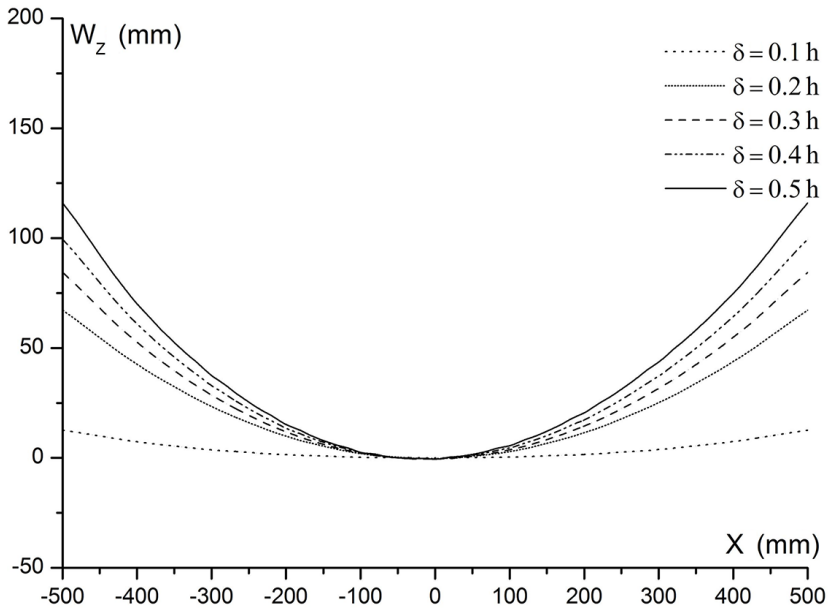

(b)

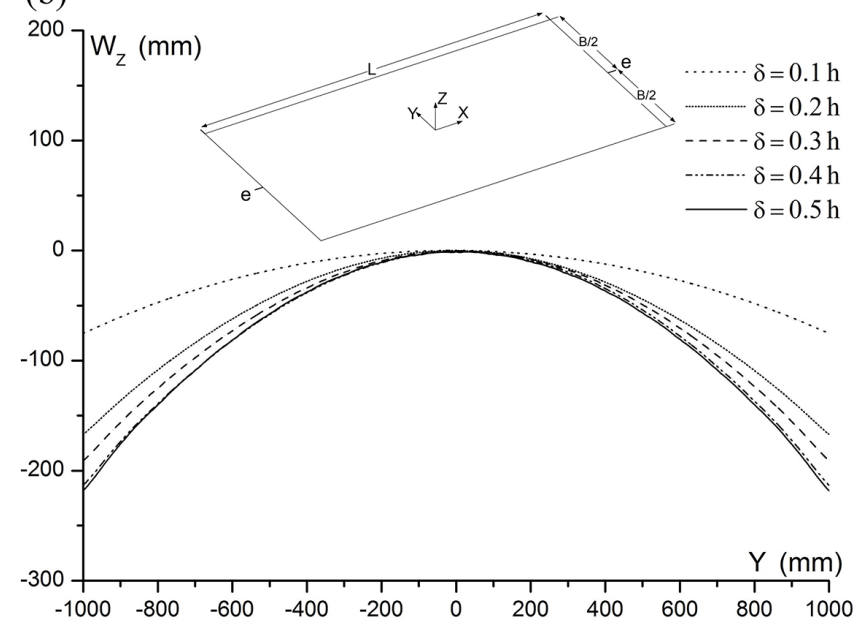

Figure 16. Impacts of roller press amount on saddle plate forming. (a) Results from lateral section. (b) Results from longitudinal section.

- Step 5: setting the press amount in database of Step 4 as the press amount of this loading path and processing all loading paths one by one.

The total calculation time consumed is approximately $40 \mathrm{~min}$ for the calculations of two loading paths. As for the overall calculation results of deformations, please refer to Fig. 15. Based on this figure, the deformation results obtained by Path 1 are exhibited as the pillow shape integrally; however, the deformation results are saddle-shaped as a whole, as far as Path 2 is concerned. As two different complex curvature shapes, they are common hull plates.

After Path 2 shown in Fig. 15b is selected for use, the press amount is set as $0.1,0.3,0.4$ or 0.5 times the plate thickness to carry out the corresponding calculations in conditions identical to those for a press amount equal to 0.2 times the plate thickness. As observed from the calculation results, the overall deformation shape still remains saddle-shaped. Deflections for the center lines (section $\mathrm{c}-\mathrm{c}$ and section $\mathrm{e}-\mathrm{e}$ ) of edges along the $x$ - and $y$-axes are shown in Fig. 16. As seen in the figure, as the press amount increases, the deflections of the saddle shape along the $x$ and $y$ directions also increase.
During practical forming loading, Eqs. (2) and (3) are usually adopted to form in-plane and bending strain distribution diagrams with the aim of more conveniently searching for path rules constituted by strain distributions in the case that key point (i) is implemented. In addition, in-plane or bending strain can be respectively applied on the pressed plate in line with a definite principle. Such analysis with diverse strain components can be applied by using the simplified deformation simulation method; on the other hand, in-plane and bending strains can also be separately exerted. Meanwhile, owing to the features of elastic calculation, if the rolling loading path calculations or calculation of other strain components applied is carried out on any plates with an initial shape, the relevant calculation process is only a repetition of the above contents. This indicates that it is feasible for the simplified deformation simulation method to be used to calculate complex curvature forming.

With regard to the actual automated local line rolling forming process, the generation of related driving data is jointly constituted by key points (i)-(iii) described above. As approximation exists among loading paths, the loading force magnitude, material property, process parameter matching, 
etc., the generation process for ultimate driving data of the automated processing equipment must be inherent with plenty of iterative numerical simulation calculations. The previous calculation results show that the simplified deformation simulation method, which is a calculation method with powerful functions, high accuracy, and efficiency, is able to play such a critical role.

\section{Conclusion}

This study focuses on a local line rolling forming method commonly used in complex curvature plate forming in the manufacture of ships. This study determines key technologies that are adopted to form automation equipment driving data for such a forming processing method. Additionally, a three-dimensional elastoplastic finite element calculation is employed to investigate the impacts of loading by virtue of such an approach on distributions of strains generated by the pressed plate. Based on the three-dimensional elastoplastic finite element calculation, a high-precision, high-efficiency simplified deformation simulation method is further proposed to support these studies on key technologies from the perspective of strain distribution laws. The relevant conclusions can be drawn as follows:

1. Regarding the local line rolling forming method used in complex curvature plate forming in the manufacture of a ship, the strain is mainly distributed within the width between the two supports of the lower roller. Specifically, the maximum principal strain appears at the center of the width. Along the direction of roller loading, strain values generated essentially remain unchanged, except at a very small area near the initiating terminal and loading termination. Moreover, when the length of the loading line extends beyond approximately 10 times the plate thickness, such a law is under a very minor impact of the loading force, loading line length, and plate thickness.

2. Strain distribution near the intersection between loading lines conforms to the corresponding loading sequence. In contrast to the length of the pressed plate or general rolling line, the involved area or length is very small or short. Therefore, they fail to exert significant influences on the overall deformation of the pressed plate. It can be estimated that the loading sequence dependency of this contour machining method can be neglected in the case that the widths of the loading lines do not mutually overlap.

3. The simplified deformation simulation method is able to preferably reflect geometrical characteristics of such a contour machining method and substantially reduce the calculation time compared with the three-dimensional elastoplastic finite element method. In addition, the workload required by strain extraction is also low. As for multiple loading paths, the strain distributions of the corresponding technological parameters can be applied to all elements of the associated paths during processing before calculations. Subsequently, only one calculation of elasticity should be implemented. This is a simple computational method. In terms of precision, its calculation results are similar to those of the three-dimensional elastoplastic finite element calculation. Nevertheless, its computational efficiency is enormously superior to the latter.

4. The simplified deformation simulation method that adopts multiple loading paths can be used to determine the shape of the plate constituted by complex curvature.

Data availability. All datasets used in the manuscript can be requested from the corresponding author.

Competing interests. The authors declare that they have no conflict of interest.

Acknowledgements. This work was supported by grant No. 2012DFR80390 from the Ministry of Science and Technology of the People's Republic of China. The authors wish to thank Kirill Rozhdestvensky of the State Marine Technical University of St. Petersburg for useful academic and constructive advices during the project.

Edited by: Z. Gronostajski

Reviewed by: two anonymous referees

\section{References}

Hwang, S. Y., Lee, J. H., Yang, Y. S, and Yoo, M. J.: Springback adjustment for multi-point forming of thick plates in shipbuilding, Comput.-Aid. Design, 42, 1001-1012, 2010.

Keisuke, K., Nakamura, T., Aoyama, H., Matsushita, N., and Ushimaru, A.: Basic study on laser forming method for curved surfaces, in: 14th International Conference on Precision Engineering, ICPE 2012, Hyogo, Japan, 995-1000, 2012.

Liu, C. and Yao, Y. L.: FEM-Based Process Design for Laser Forming of Doubly Curved Shapes, J. Manufact. Process., 7, 109-121, 2005.

Nguyen, T. T., Yang, Y. S., Bae, K. Y., and Choi, S. N.: Prediction of deformations of steel plate by artificial neural network in forming process with induction heating, J. Mech. Sci. Technol., 23, 12111221, 2009.

Park, J., Kim, D., Hyun, C., and Shin, J.: Thermal forming automation system for curved hull plates in shipbuilding, Analysis and design, Int. J. Comput. Integr. Manufact., 29, 287-297, $2016 \mathrm{a}$.

Park, J., Kim, D., Mun, S., Kwon, K., and Lee, J.: Automated thermal forming of curved plates in shipbuilding: system development and validation, Int. J. Comput. Integr. Manufact., 29, 1128$1145,2016 b$. 
Shaohui, W., Zhongyi, C., Mingzhe, L., and Yingwu, L.: Numerical simulation on the local stress and local deformation in multipoint stretch forming process, I. J. Adv. Manufact. Technol., 9, 901-911, 2012.

Shi, Y. J., Yi, P., and Hu, J.: Effects of process parameters on forming accuracy for the case of a laser formed metal plate, Lasers Eng., 26, 295-310, 2013.

Shim, D. S., Yang, D. Y., Lee, D. J., and Han, M. S.: Investigation into the process design for the manufacture of doubly curved plates in the incremental roll forming process and its experimental verification, in: 10th International Conference on Technology of Plasticity, ICTP 2011, Aachen, Germany, 373-378, 2011.

Ueda, Y., Murakawa, H., Rashwan, A. M., Okumoto, Y., and Kamichika, R.: Development of computer-aided process planning system for plate bending by line heating (report 1): Relation between the Final Form of Plate and the Inherent Strain, Trans. JWRI, 20, 275-285, 1991.

Ueda, Y., Murakawa, H., Rashwan, A. M., Kamichika, R., Ishiyama, M., and Ogawa, J.: Development of computer-aided process planning system for plate bending by line heating (Report 4): Decision Making on Heating Conditions, Location and Direction, Trans. JWRI, 22, 305-313, 1993.

Ueda, Y., Murakawa, H., Mohamed, A., Okumoto, Y., and Kamichika, R.: Development of computer-aided process planning system for plate bending by line heating (report 2). Practice for plate bending in shipyard viewed from aspect of inherent strain, Trans. Jwri, 21, 123-133, 1994a.
Ueda, Y., Murakawa, H., Mohamed, A. M., Neki, I., Kamichika, R., Ishiyama, M., and Ogawa, J.: Development of computer-aided process planning system for plate bending by line heating (report 3): Relation between heating condition and deformation, Trans. Jwri, 22, 145-156, 1994b.

Wu, Y.: Analysis on Springback of High Strength Steel Plate with Experiments and Finite Element Simulation, MS Thesis, Huazhong University of Science \& Technology, Huazhong, 2014.

Yoon, S. J. and Yang, D. Y.: Development of a Highly Flexible Incremental Roll Forming Process for the Manufacture of a Doubly Curved Sheet Metal, CIRP Ann. Manufact. Technol., 52, 201204, 2003.

Yoshihiko, T., Morinobu, I., and Hiroyuki, S.: "IHIMU- $\alpha$ " a fully automated steel plate bending system for shipbuilding, J. IHI Technol., 51, 24-29, 2011.

You, W., Mingzhe, L., Daming, W., and Anyuan, W.: Modeling and numerical simulation of multi-gripper flexible stretch forming process, Int. J. Adv. Manufact. Technol., 73, 279-288, 2014. 